\title{
Dissecting immune cell stat regulation network reveals biomarkers to predict ICB therapy responders in melanoma
}

Jingwen Wang ${ }^{\dagger}$, Feng $\mathrm{Li}^{\dagger}$, Yanjun $\mathrm{Xu}^{\dagger}$, Xuan Zheng, Chunlong Zhang, Congxue Hu, Yingqi Xu, Wanqi Mi, Xia Li* and Yunpeng Zhang*

\begin{abstract}
Background: Immunotherapy is a revolutionary strategy in cancer therapy, but the resistance of which is one of the important challenges. Detecting the regulation of immune cells and biomarkers concerning immune checkpoint blockade (ICB) therapy is of great significance.

Methods: Here, we firstly constructed regulation networks for 11 immune cell clusters by integrating biological pathway data and single cell sequencing data in metastatic melanoma with or without ICB therapy. We then dissected these regulation networks and identified differently expressed genes between responders and non-responders. Finally, we trained and validated a logistic regression model based on ligands and receptors in the regulation network to predict ICB therapy response.

Results: We discovered the regulation of genes across eleven immune cell stats. Functional analysis indicated that these stat-specific networks consensually enriched in immune response corrected pathways and highlighted antigen processing and presentation as a core pathway in immune cell regulation. Furthermore, some famous ligands like SIRPA, ITGAM, CD247and receptors like CD14, IL2 and HLA-G were differently expressed between cells of responders and non-responders. A predictive model of gene sets containing ligands and receptors performed accuracy prediction with AUCs above 0.7 in a validation dataset suggesting that they may be server as biomarkers for predicting immunotherapy response.
\end{abstract}

Conclusions: In summary, our study presented the gene-gene regulation landscape across 11 immune cell clusters and analysis of these networks revealed several important aspects and immunotherapy response biomarkers, which may provide novel insights into immune related mechanisms and immunotherapy response prediction.

Keywords: Immune single cells, Regulation networks, Immunotherapy

\section{Background}

The majority of skin cancer-relevant deaths are accounted by melanoma $[1,2]$. Only $23 \%$ of metastatic (stage IV) melanoma patients survived over 5 years after diagnosis [1]. Immunotherapy raised a revolutionary weapon

*Correspondence: lixia@hrbmu.edu.cn; zhangyp@hrbmu.edu.cn ${ }^{\dagger}$ Jingwen Wang, Feng Li and Yanjun Xu contributed equally to this work College of Bioinformatics Science and Technology, Harbin Medical University, Harbin 150081, China against cancer [3-5]. Notably, anti-CTLA4 and anti-PD-1 inhibitors, ipilimumab and nivolumab, have achieved great increase in clinical benefit for carcinomas like metastatic melanoma [6-8]. Even though oncology is being revolved by the remarkable success of ICB therapies, the majority of patients received immune checkpoint blockade (ICB) therapies unfortunately do not benefit from the treatment [9-12]. Primary and acquired resistance also

(c) The Author(s) 2021. This article is licensed under a Creative Commons Attribution 4.0 International License, which permits use, sharing, adaptation, distribution and reproduction in any medium or format, as long as you give appropriate credit to the original author(s) and the source, provide a link to the Creative Commons licence, and indicate if changes were made. The images or other third party material in this article are included in the article's Creative Commons licence, unless indicated otherwise in a credit line to the material. If material is not included in the article's Creative Commons licence and your intended use is not permitted by statutory regulation or exceeds the permitted use, you will need to obtain permission directly from the copyright holder. To view a copy of this licence, visit http://creativeco mmons.org/licenses/by/4.0/. The Creative Commons Public Domain Dedication waiver (http://creativecommons.org/publicdomain/ zero/1.0/) applies to the data made available in this article, unless otherwise stated in a credit line to the data. 
obstruct long-term curative-effect in patients with metastatic melanoma [9].

ICB therapy provided a great sense to investigate immune cell interactions of melanoma [13]. It is an urgent need to practice regulation analysis and data mining among immune cells under context of on- or posttherapy in melanomas. By profiling of single immune cells in baseline and on- or post-therapy samples in melanoma patients treated with checkpoint therapy, Hacohen et al. has defined 11 CD45+immune cell stats which were associated with response to ICB therapy in metastatic melanoma [14]. These ICB therapy related CD45+immune cell stats enable construction of regulation networks for different immune cell stats.

Currently, primary biomarkers of ICB therapies such as tumor mutational burden (TMB) and programmed death ligand 1 (PD-L1) expression $[7,15]$ have performed rough immunotherapy selection, and new biomarkers (e.g., eosinophilic count) show associations toward poor or longer survival [16]. For predicting of response to therapy, there are several studies attempt to inquire into alterations in expression of the PD-1/PD-L1 immune inhibitory axis or tumor microenvironment in patients with melanoma $[8,15]$. Also, some studies focused on heterogeneities of individual cells by single-cell RNA sequencing $[14,17]$. However, these researches have thus far provided only a limited understanding for immunotherapy response. Novel potential biomarkers and prediction models are still urgently needed.

Here, we constructed regulation networks based on specific immune cell stat, dissected transcriptome features of multiple single-cell cluster binding pathways and investigated predictive capability of single-cell-based network model. Our observation showed that ligands and receptors in immune cell related regulation networks have selective power of therapy response in patients receiving ICB inhibitors.

\section{Materials and methods Materials}

$C D 45+$ single cell sequencing Immune cell high throughput sequencing matrix of melanoma we used in this study was accessible in GEO database (GSE120575) which profiled 16,291 CD45+ immune cells from 48 tumor samples [14].

Pathways As reference pathways of our networks, BioPAX [18] level 3 integrated pathways were downloaded from https://www.pathwaycommons.org/archives/PC2/ v10/. The common pathways contain 2,374,707 interactions of 32,875 participants integrated as 13 types (e.g., controls-expression-of, in-complex-with).

mRNA sequencing of bulk tumor samples mRNA expression of 56 MAGE-A3 checkpoint inhibitor treated melanomas was downloaded from GEO database (GSE35640). And twenty-two of these patients had complete or partial response to immunotherapy [19].

Others Homo species transcription factors (TF) were downloaded from AnimalTFDB [20] (http://bioinfo.life. hust.edu.cn/AnimalTFDB/\#!/). Paired ligands and receptors were obtained from FANTOM [21] (http://fantom.gsc. riken.jp/5/suppl/Ramilowski_et_al_2015/) database.

\section{Methods \\ Integrating networks of melanoma immune cells}

Each of the 16,291 immune cells was previously assigned into one of 11 unsupervised clusters according to the study of Sade-Feldman et al. [14]. Sade-Feldman M et al. applied the k-means algorithm to classify CD45+immune cells. They used all genes with variance $>6$ (about 4000 genes) to identify the unsupervised clustering of immune cells. They applied the k-means algorithm testing $\mathrm{k}=3 \ldots 15$, and when $k=11$, the clusters are most robust based on the 100 iterations in which they removed $10 \%$ of the cells randomly. Among those 11 clusters, $\mathrm{C} 1$ and $\mathrm{C} 2$ tend to be enriched by $\mathrm{B}$ cells, $\mathrm{C} 3$ and $\mathrm{C} 4$ are myeloid clusters and $\mathrm{C} 6$ and $\mathrm{C} 9$ are $\mathrm{T}$ cell clusters [14]. For each cluster, we constructed an immune cell cluster specific network considering three following aspects, in other words, two genes are considered to have an interaction relationship in our network and need to meet the following three conditions, a) each of the two genes must transcript in more than $1 \%$ immunity cells; b) there must be an interaction type in BioPAX pathways between them; c) co-expressed p-value (spearman correlation test) of these two genes must under 0.05 (Additional file 9: Figure S1).

We combined 11 immunity cell cluster specific networks (Additional file 8) into regulation networks and annotated them with TFs, ligands and receptors (Fig. 1, Additional file 9: Figure S1).

\section{Topological properties evaluation of cell cluster specific networks and regulation networks}

According to Barabási et al. [22, 23] and Zhang et al. [24], real biological gene interaction lies in scale-free networks, and degree distributions of genes in the network should comply with power law distribution (Eq. 1). We fit liner model for logarithm transformed gene degrees $(\mathrm{X})$ and their distributions $(\mathrm{Y})$ in a derived formula (Eq. 2) where a and $b$ are coefficients to fit.

$$
\begin{aligned}
& Y=a X^{-b} \\
& \log _{10} Y=-b \log _{10} X+\log _{10} a
\end{aligned}
$$




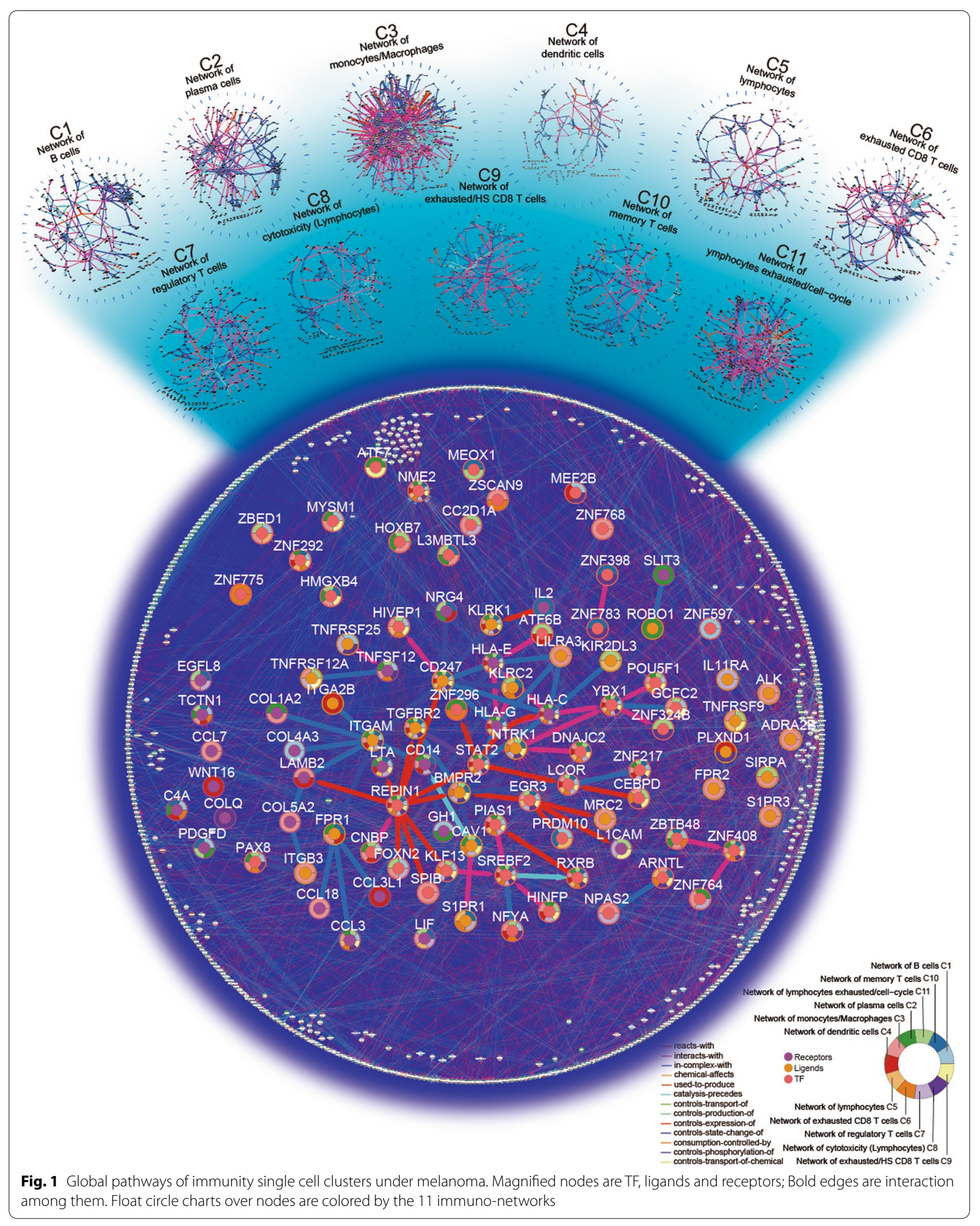




\section{Identifying differently expressed genes}

We divided cells into four groups: (1) cells from pretreatment responders; (2) cells from pre-treatment nonresponders; (3) cells from on-treatment responders; (4) cells from on-treatment non-responders. Then we calculated two measurements for responder and nonresponder groups to access different expression-fold changes and Wilcox test $\mathrm{p}$ values. Finally, we used twofold and 0.05 significance as thresholds to define differently expressed genes.

\section{Immunotherapy decision classification based on regulation networks}

We investigated the predictive power of regulation networks in immunotherapy resistance by following steps:

(1) fit logistic models-ligands and receptors in regulation networks were used as origin variables for model fitting in train data sets (half of GSE120575 samples selected randomly). We used "glm" function in R package "stats" to fit logistic models;

(2) choose variables-formula-based model auto-selection were applied to select a subset of ligands and receptors along with their coefficients that can optimally guide therapy outcomes. We used "step" function to auto-select the logistic models and "predict" function to access prediction power of selected model;

(3) test precision-variable sets we chosen in step 3 were tested in another half of GSE120575 samples. The "predict" function was used to calculate AUC in test data sets;

(4) repeat circulation-step 1 to 3 were repeated 10,000 times.

Finally, the AUCs in above steps were used as standards to access prediction power of ligands and receptors in regulation networks.

\section{Results}

Regulation networks of immunity cell stats in melanoma

Tumor cell-intrinsic heterogeneity shapes the immune cell infiltration and influences the outcome of immunotherapy [25], and various metabolic pathways orchestrate the behavior of tumor-infiltrating immune cells, which are related to enhancing of antitumor immunity and immunotherapy [26]. Moreover, distinct CD45+ cell clusters revealed by single-cell RNA-seq were associated with clinical outcome of ICB therapies and reflected by different identifiers [14]. Thus, co-expression of pathway genes in multiple cell stats could offer cellular immunity regulation. Herein, we constructed immunity networks based on BioPAX pathways for previously defined
CD45+ cell clusters [14] and combined them into regulation networks (Fig. 1, Additional file 9: Figure S2, Method Details). We fitted power law models for degree distributions of cell cluster specific networks and regulation networks (Additional file 9: Figure S3, Method Details), and the $\mathrm{R}$ squares greater than 0.88 all over these models. Immune networks and regulation networks constructed by our pipeline are scale free and similar to the real biological networks.

In sum, seven hundred and ninety genes, including 26 receptors, 26 ligands, and $47 \mathrm{TFs}$, and 3048 interactions were recognized by the regulation networks. Notably, IL-2 receptor is a T cell stimulating cytokine [27], and it not only drives the expansion of $\mathrm{T}$ cells and the contraction phase of immune response [28], but also has an effect on cancer stem cells $[29,30]$. Most importantly, low dose IL2 combined with other immunotherapy demonstrated benefit in patients with metastatic melanoma [31]. In C10, a memory T cell cluster, IL2 controls expression of KLRK1 which controls state change of ITGAM through CD247 (in complex with HLA-C, HLA-E and HLA-G in all 11 cell clusters). IL2 controlling ITGAM, which in complex with CD14 (IL2, ITGAM, CD14 are cell surface markers) [32], indicted that our framework efficiently highlighted regulation flows of immunity system in distinct and common immune cell stats. Construction of immune cell stat specific pathways could offer contributions for immunology and ontology, including immune therapy response related researches.

\section{Divergence regulation of overlap genes was revealed by network comparative analysis among multiple networks}

Since our study highlighted different interaction flows of cluster specific networks, we accessed expression of genes and interaction pairs in distinct immune cell stats. There are several genes (86) such as major histocompatibility complex (HLA-C, HLA-DMA, HLA-DMB, HLA-DRA, HLA-E, HLA-G and HLA-H), CD247, cyclin dependent kinase (CDK11B), casein kinase 2 (CSNK2A1 and CSNK2B), FGFR1 oncogene partner (FGFR1OP) etc. that were activated in all 11 predefined $T$ cell stats (Fig. 2a). However, consensus interactions among those common genes were partly observed. On the contrary, obvious inconsistent interaction pattern among common genes were discovered in $\mathrm{B}$ cell clusters $(\mathrm{C} 1, \mathrm{C} 2)$, myeloid clusters $(\mathrm{C} 3, \mathrm{C} 4)$ and $\mathrm{CD} 8 \mathrm{~T}$ cell clusters $(\mathrm{C} 6, \mathrm{C} 9)([14]$, Fig. 2b, Additional file 9: Figure S4). Even though identifiers of distinct immune cell stat differ from the others, there are common genes, which exercise functions through different flows, that were activated in multinetworks. For instance, mechanistic target of rapamycin kinase (MTOR) state change was controlled by HLA-G 


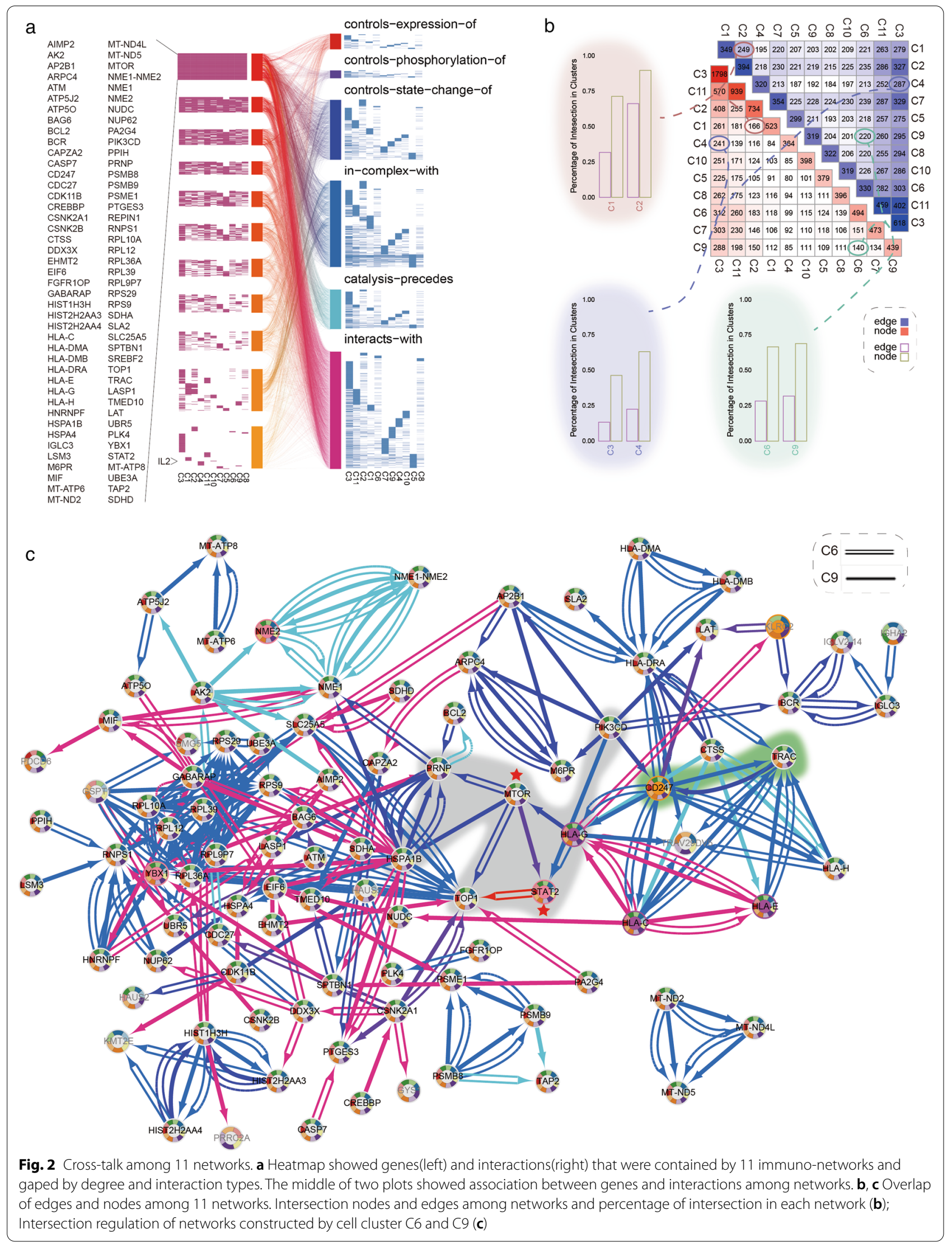


in C9 but not in C6, and MTOR controls state change of HSPA1B in C9, TOP1 and PRNP in C6 (Fig. 2c). STAT2 interacts with different genes in C6 (HLA-G) and C9 (MTOR, PIK3CD). Moreover, a specific duplex interaction was observed in C9 (TRAC controls state change of CD247, CD247 in-complex-with TRAC, Fig. 2c). These results provide further evidence for our explanation that common genes from different cell stats can activate in variety ways in these cells.

Interleukin-2 (IL-2) antigen stimulates memory $\mathrm{CD} 8(+) \mathrm{T}$ cells production, and high relative IL-2 production in $\mathrm{T}$ cells of melanoma tend to perform memory $\mathrm{CD} 8(+) \mathrm{T}$ cells phenotype and superior proliferative capacity compared to cells with low IL-2 production [33]. In our research, IL2 and LIME1 specifically activated in a memory $\mathrm{T}$ cell cluster (C10). We also calculated different expression of IL2 in C10. The expression levels of IL2 gene is significantly expressed higher in cells from responders than cells from non-responders of ontreatment patients. Furthermore, several genes activated distinctly in different networks. For instance, PVRIG specialized in lymphocytes (C5), AGER, BHLHB9, CDK3, GNG8, IL11RA, PKIA, USP50 in regulatory T cells (C7). ARHGAP19, FNIP1 in exhausted/HS CD8 T cells (C9) and DECR2, SESTD1, ZNF775 in exhausted CD8 T cells (C6) etc. Our framework discovered different performance of immune related genes in cell cluster specific networks, which may lead to a functional nuance.

\section{Consensus functions across immune cell regulation networks}

To investigate functional relevance of cell cluster specific networks, we employed online pipeline metascape (http://metascape.org/gp/index.html) for enrichment analysis. We found that gene sets from plural networks enriched in consensus functions, for example, metabolism of RNA, antigen processing and presentation, cell cycle and herpes simplex infection, regardless of the different activation flows they presented (Fig. 3). Significantly, all networks partly enriched in several subterms of vital functions like $\mathrm{T}$ cell activation, cell cycle, cellular responses to stress, apoptotic signaling pathway, cytokine-mediate signaling pathway, herpes simplex infection, metabolism of RNA [34-38]. As for some functions, such as negative regulation of immune system process, were enriched by all networks but in different sub-terms. In addition, exhausted CD8 $\mathrm{T}$ cell cluster (C6) and lymphocytes exhausted/cell-cycle (C11) cluster significantly enriched in regulation of complement activation overall situation, yet regulation of complement activation was not enriched by monocytes/macrophage cluster(C3), cytotoxicity (lymphocytes) cluster (C8) and exhausted/HS CD8 T cell cluster (C9). Several sub-terms of negative regulation of immune system process were enriched by different networks despite all eleven networks were enriched in negative regulation of immune system process by a significant level. Computation of enriched function in immune cells could perform a systemic insight to understand mechanism of immune cell interaction.

\section{A core pathway: antigen processing and presentation}

We discovered that shared topology of 11 networks covered two major histocompatibility complex unions (HLAC, HLA-E, HLA-G; HLA-DMA, HLA-DRA, HLA-DMB), one ribosomal protein union (RPL10A, RPL9P7, RPL39, RPL12, RPL36A, RPS29, RPS9), one mitochondrially encoded NADH: ubiquinone oxidoreductase core subunit (MT-ND2, MT-ND4L, MT-ND5), one NME/NM23 nucleoside diphosphate kinase union (NME1, NME1NME2) and one proteasome and proteasome activator subunit (PSME1, PSMB8, PSMB9) (Fig. 4a). Enrichment analysis of these shared topologic structures showed concurrent functions like regulation of expression of SLITs and ROBOs, antigen processing and presentation of exogenous peptide antigen, antigen processing and presentation peptide antigen assembly with MHC class II protein complex and neutrophil deregulation (Fig. 4b, c). Especially, subunits of the shared structures lie in two flows of antigen processing and presentation, MHC I and II pathways ([39], Fig. 4d), which play upstream roles of CD8 T cell killing target cells, regulation of NK cell activity (MHC I) and CD4 T cell cytokine production and activation of other immune cells (MHC II). According to Gene Ontology [40] enrichment analysis executed by metascape, three antigen related functions are interconnected among them, and the most significant enrichment is antigen processing and presentation.

\section{Key genes from networks were related with immunotherapy response at single cell level}

Prior knowledge showed that a large number of genes appeared relevance with not only cancer occurrence but also treatment response, and these appearances came up in the single cell level as well [14, 19, 41-43]. Expectably, there were a batch of genes in our regulation networks which were differently expressed between responders and non-responders in both periods of treatment (pre- and on-treatment) regardless of immune cell stats (Fig. 5a). Functional analysis showed that genes expressed higher in non-responders from on-treatment samples were enriched in cytokine-mediated singling pathway, transmembrane receptor protein tyrosine kinase signaling pathway and so on, yet differently expressed genes from pre-treatment samples were enriched in immune response-activating signal transduction, response to 


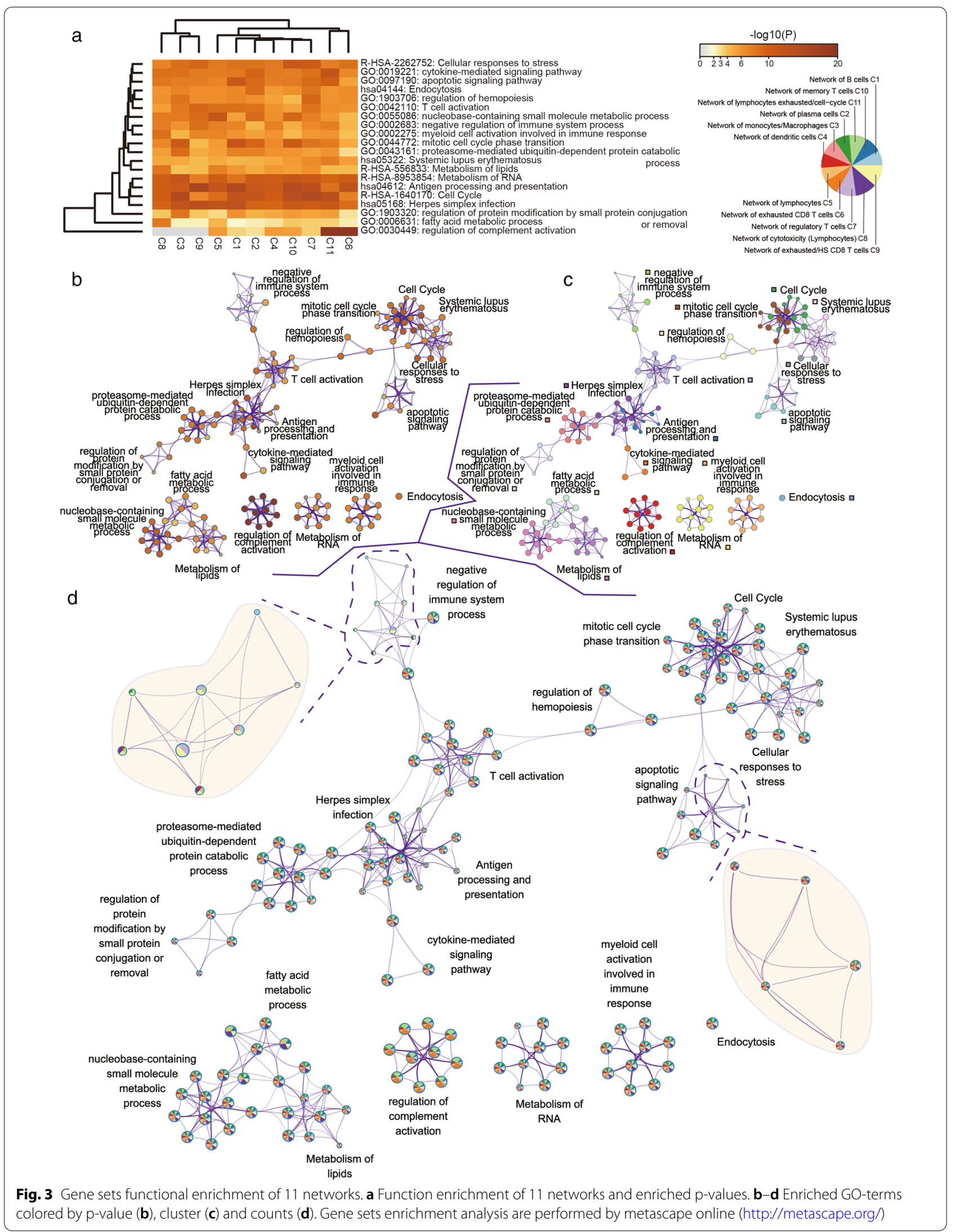




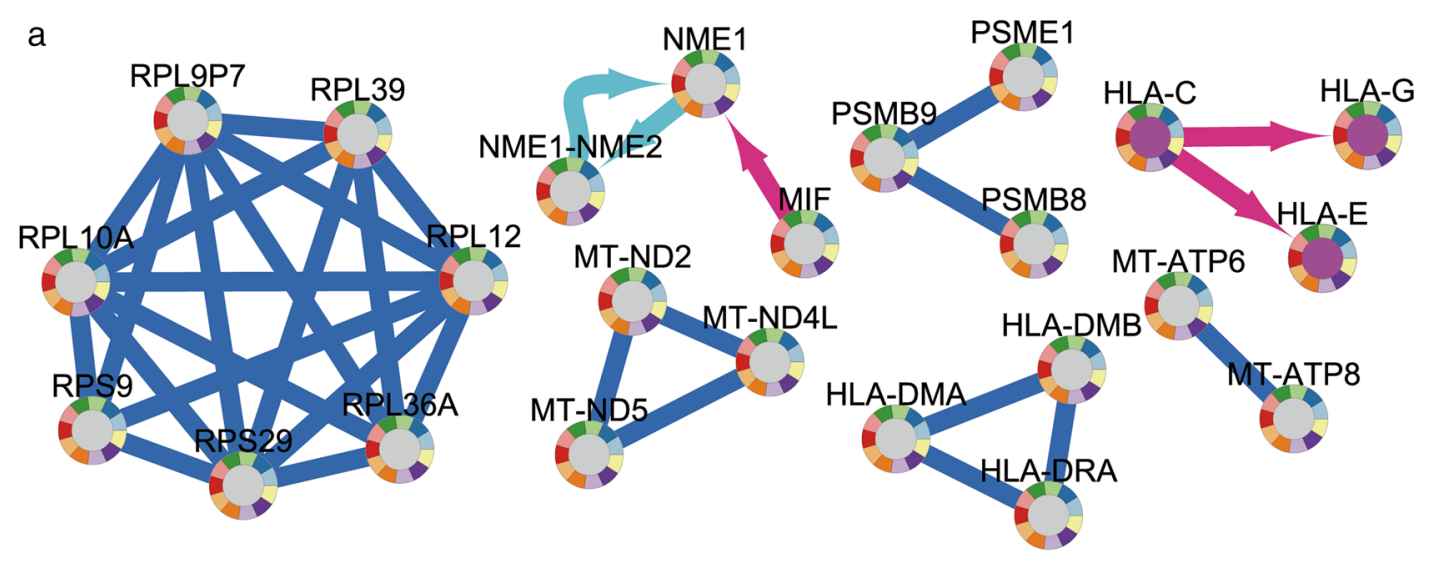

b

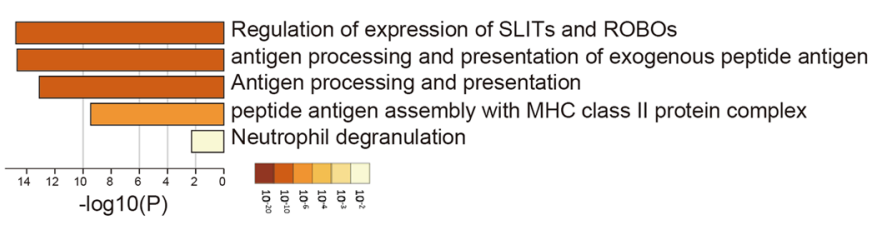

d Antigen Processing And Presentation
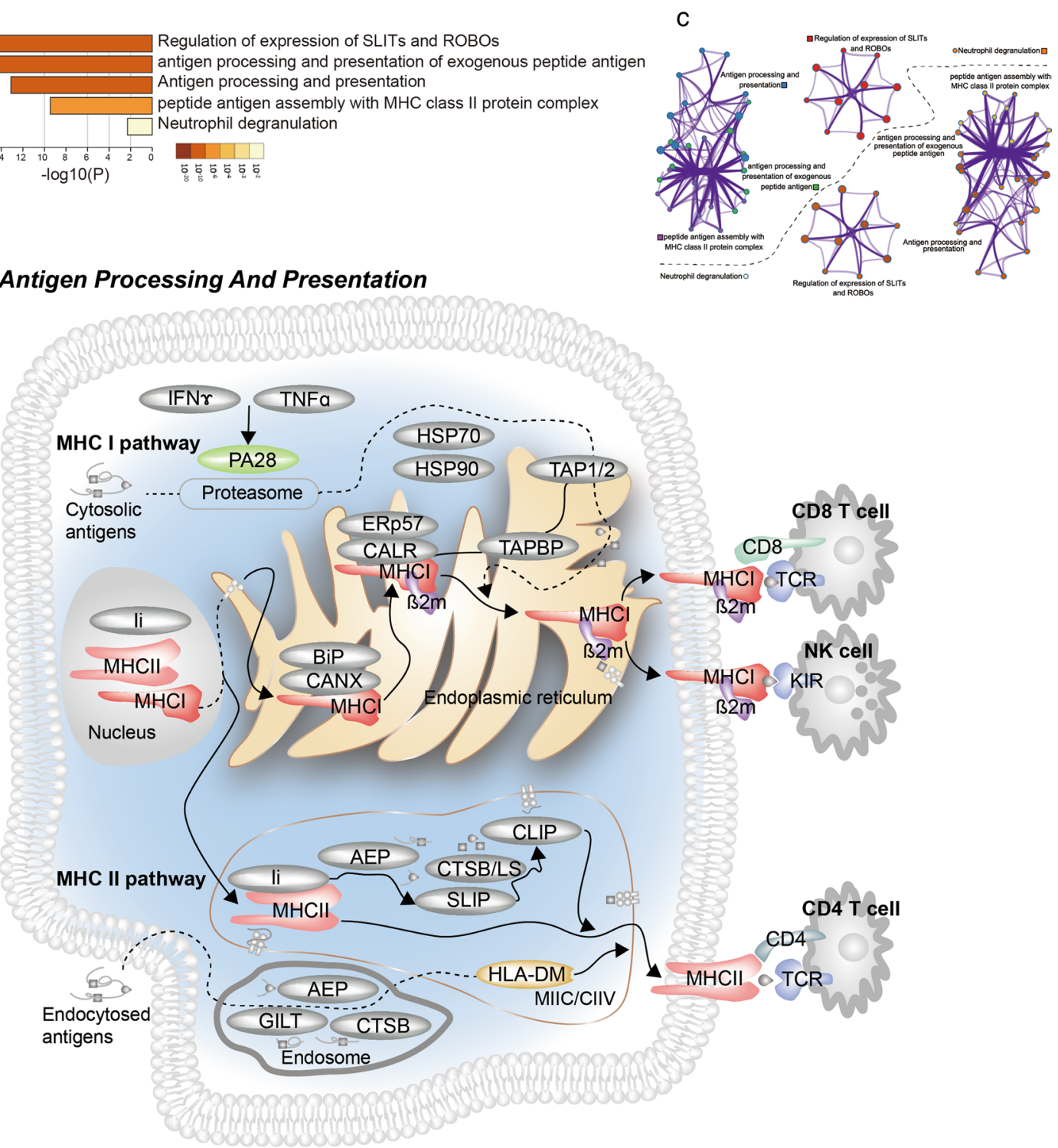

Fig. 4 Shared sub-networks of 11 networks. a Shared interactions of 11 networks. $\mathbf{b}$ and $\mathbf{c}$ Function enrichment of shared genes. Gene sets enrichment analysis are performed by metascape online (http://metascape.org/). d A core pathway: Antigen Processing and Presentation. Colored nodes are enriched proteins 


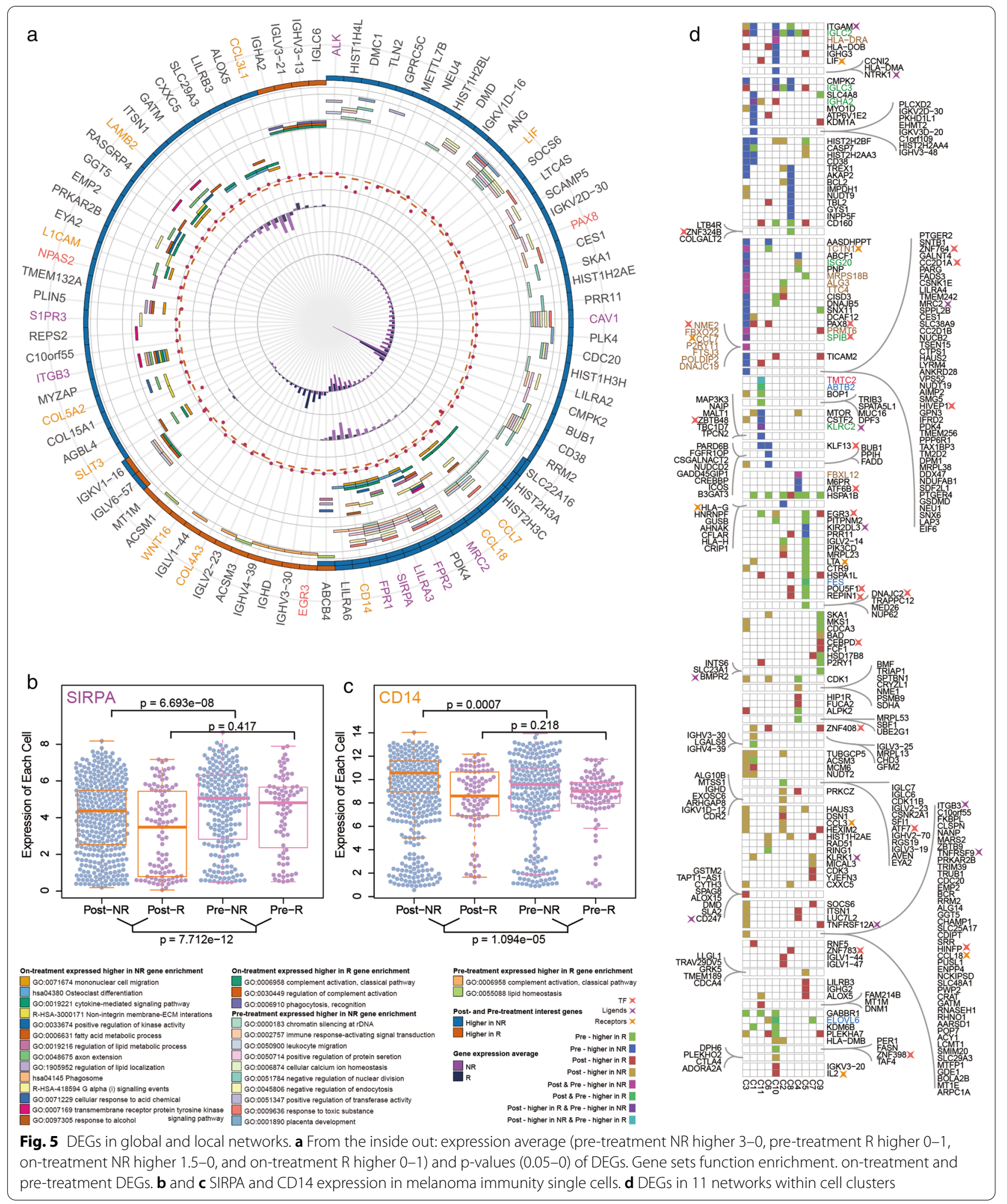


toxic substance etc. Interestingly, several ligands and receptors differently expressed between responders and non-responders in pre- or (and) on-treatment samples, especially for a higher-in-non-responder set (CCL7, CCL18, MRC2, FPR2, LILRA3, SIRPA and CD14). Moreover, we detected a ligand, SIRPA, significantly expressed higher in non-responders and in pre-treatment samples. Consequently, SIRPA may play a resistance role in immunotherapy [44-46], and CAV1, SIRPA, CD14 were expressed higher in non-responders, may potentially support drug antagonism ([44-49], Fig. 5b, c).

Although we detected dysregulated genes in the single cell level, it still needs further inspection to uncover transcriptome changes in immune cell stats. Therefore, we next assessed differently expressed genes from cluster specific networks in corresponding CD45+ immune cell stats (Fig. 5d, Additional file 9: Figure S5-6). Our research suggested that ligand integrin subunit alpha M (ITGAM) was significantly upregulated in C3 from non-responders of on-treatment patients, in $\mathrm{C} 1$ and C10 from non-responders of pre-treatment patients, as well as in C8 from responders of pre-treatment patients. Regulating cell fate, ITGAM was dysregulated in multiple cell types ( $\mathrm{T}$ cells, B cells et.), which may contribute to tumor cell survival in immunotherapy [50]. Ligand NTRK1, which expressed higher in pre-treatment nonresponders, was dysregulated in C10, and IL2, a type I cytokine which can be associated with durable regression in metastatic melanoma and renal cell carcinoma [51], also was dysregulated in and only in C10 but expressed higher in on-treatment responders. Two major histocompatibility complexes, HLA-G and HLA-H, expressed higher in $\mathrm{C} 2$ from pre-treatment non-responders. Another major histocompatibility complex, HLA-DMB, expressed higher in both $\mathrm{C} 2$ and $\mathrm{C} 10$ in non-responders from on-treatment samples. Other famous genes, such as tumor necrosis factor receptor superfamily member 9 (TNFRSF9) and CD247 ligands, were also upregulated in C3 from (non-)responders of on-treatment patients, and another tumor necrosis factor receptor superfamily member (TNFRSF12A) was dysregulated in both C3 and C9. Thus, we have reason to suppose that some ligands and receptors may propose a new insight to understand drug sensitivity and resistance, as well as immunotherapy response.

\section{Ligands and receptors of regulation networks showed robust selective power in immunotherapy response}

Some receptors can mediate functions of immune cells through distinct signaling pathways [52]. Changing of these receptors and corresponding ligands may lead unexpected immunotherapy outcomes. Our research also observed massive changes of gene expression of these proteins. Hence, we applied logistic regression model to select contribution features from ligands and receptors of regulation networks and access immunotherapy precision of featured gene sets. In the test data sets, medium AUC of 10,000 random is 0.7143 , and most of the test AUCs are among 0.65 and 0.8 (Fig. 6a). Eventually, we identified 17 gene sets that associated with immunotherapy response. In an independent validation, GSE35640, all 17 gene sets performed AUC greater than 0.7 (Fig. $6 \mathrm{~b}$ ), which suggests robust selective power of ligands and receptors of regulation networks for immunotherapy response. This completed key gene analysis by providing gene sets and their scores which were able to construct classification of therapy response.

Several TNF receptor superfamily members were integral to the immune-response regulation by enhancing T-cell growth and dendritic-cell function. These proteins related to modulation of cellular functions, proliferation, survival or deaths [53]. Moreover, TNF receptors controlling TNF receptor signaling, which plays its role in inflammation and cell death, could determine the cellular fate [54]. Especially, TNFRSF12A (also known as TWEAK receptor, Fn14, or CD266) correlated with integrin $\beta 3$ expression, which drives Glut3 expression, is associated with clinical outcome and tend to be responsible for inducing cachexia in tumors $[55,56]$. We identified one TNF receptor leaded gene set, consisting of TNF receptor superfamily member 9/25/12A (TNFRSF9, TNFRSF25 and TNFRSF12A), sphingosine-1-phosphate receptor 1 (S1PR1), C-C motif chemokine ligand 3 (CCL3), caveolin 1 (CAV1) and major histocompatibility complex, class I, G (HLA-G), that predicted immunotherapy response with AUC up to 0.7433 in independent validation and 0.8824 in GSE12575 (Fig. 6c). Besides, CAV1 catalysis preceding of resistance related gene CD14 [49, 57], as well as supported a firm set predicting immunotherapy response (0.7045 in GSE35640, 0.871 in GSE120575) with CD247, HLA-C, HLA-E, ITGAM and CD14. Thus, TNFRSF12A, which works for 11 of 17 selected gene sets, cooperating with CAV1 and other ligands and receptors are key factors in immunity regulation and flexible immunotherapy response (Fig. 6d).

Additionally, other gene sets with major histocompatibility complexes (HLA-C, HLA-E, HLA-G), TNF receptors, ITGAM, CD247 and CD14 performed acceptable precision with AUC around 0.72 in the independent datasets and above 0.85 in GSE12575 as well. Furthermore, ITGAM, which regulates cell fate [50], also works 11 of 17 selected genes sets and HLA-C works in 9 genes sets (Fig. 6e). These genes not only activate in majority immune cells but also have a quality for prediction of immunotherapy response. 


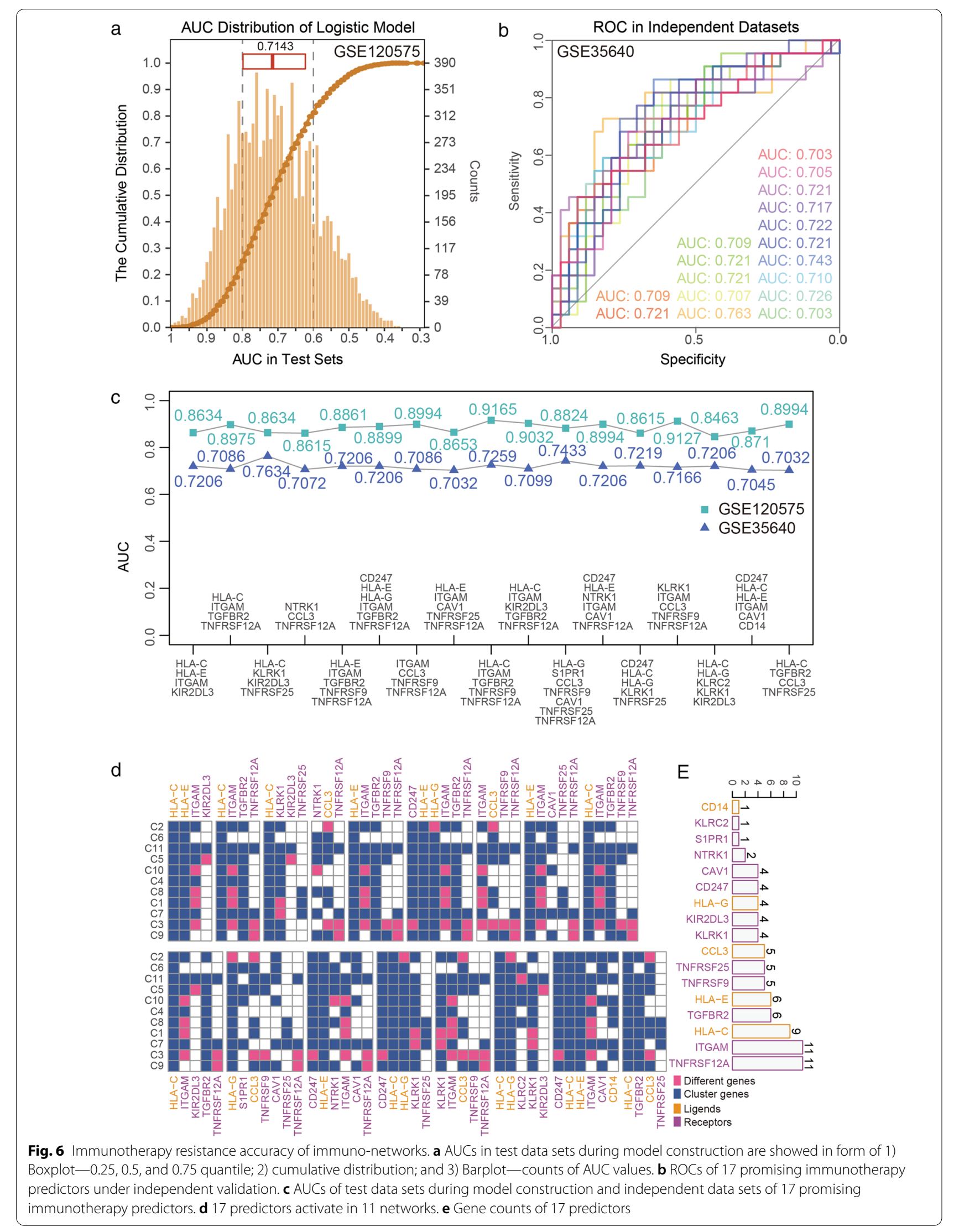




\section{Discussion}

Great progress has been achieved in ICB therapy, yet therapy resistance must be considered in an actual treatment process. Consequently, it is important to investigate biomarkers of immunotherapy especially in single cell level. In this study, we constructed regulation networks across immune single cell types and established that differently expressed genes between cells from responders and non-responders. Some differently expressed genes coincided with ligands and receptors in immune cell specific pathways. Further analysis of ligands and receptors in regulation networks proposed prediction biomarkers for inhibitor response classifier in metastatic melanoma with ICB therapy. We trained logistics regression models to test prediction accuracy of biomarkers for immunotherapy response. Our results suggested 17 gene sets which could be useful for prediction of ICB therapy.

We found out that metabolism of RNA, antigen processing and presentation, cell cycle and herpes simplex infection networks were generally activated in all immune cell clusters but with different interaction flows. We also found that different sub-terms of negative regulation of immune system process were enriched by variety cell cluster specific networks even all the cell stats activated negative regulation of immune system process. Notably, we discovered a common topologic structure of all immune cell specific networks performing antigen processing and presentation function, which connected with antigen processing and presentation of exogenous peptide antigen and peptide antigen assembly with MHC class II protein complex. These three terms were essential for endogenous simulated immunity defense with cell surface MHC molecular carry and display viral peptides [58]. It requires an army of genes to coordinate for immune response and raise a weapon against tumor. MHC class I and class II molecules played a global relative role in presentation and processing of the antigen with its high polymorphic [59]. For MHC class I, we detected that HLA-C respectively interacted with HLA-G and HLA-E. And for MHC class II, we found a triple complex relationship among HLA-DMA, HLADMB and HLA-DRA. These results indicated that antigen processing and presentation may be a core functional region in immune cell regulation and proposed further explanation of immune response in ICB therapy.

We further revealed some key factors such as SIRPA, CD14, IL2, ITGAM and CD247 (differentially expressed between cells of responders and non-responders). These ligands and receptors tend to be associated with drug sensitivity and resistance, as well as immunotherapy response. Especially, IL2 controls expression of KLRK1 which controls state change of ITGAM through CD247. IL2 controlling ITGAM, which in complex with CD14 (both are cell surface markers, [32]), are specific in C10 and this type I cytokine can be associated with durable regression in metastatic melanoma and renal cell carcinoma [51]. In this study, biomarker gene sets detected by $\mathrm{CD} 45+$ immune single cells showed robust perspective power in ICB therapy response. Our results suggested that TNF receptors, MHC molecules, ITGAM, CD247 or CD14 leading gene sets can preciously distinguish the responders for non-responders in patients with melanoma that received ICB therapies. The accuracy of immune cell regulation network-based model may provide helpful guidance for precision medicine, as well as new understanding of immunotherapy response. Specifically, SIRPA and CD14 were upregulated in nonresponders of both pre- and on-treatment patients. They both regulate TRIM27, and CD14 which contribute as a member of prediction gene sets in the logistics models. CD14, ITGAM, CD247 and MHC molecules not only significantly dysregulated between the responders and non-responders of patients with melanoma but also presented accuracy prediction of immunotherapy response. Several famous genes like MHC molecules (HLA-C/E/G), TNF receptor superfamily members (TNFRSF9/12A/25), ITGAM, CD14, CCL3 and CAV1 all performed dysregulation between responders and non-responders in the global or immune cell stat specific context, and they also provided great independent cooperation in prediction of immunotherapy response.

We identified 17 gene sets that associated with immunotherapy response. In an independent validation, GSE35640, all 17 gene sets performed AUC greater than 0.7 . We compared 17 predictors identified by our analysis to ICB response biomarkers (PD-L1 and IFNG) which were widely used by clinical trials [60-63]. Our results showed that AUC of PD-L1 expression is 0.69 and AUC of IFNG expression is 0.75 (Additional file 9: Figure S7). Our results indicated that using ligands and receptors in the regulation networks to train decision models could provide a brand-new view for immunotherapy response, and these models could be potential guidance for precision medicine. We also tried to compare our predictor with TIDE [64]. However, the TIDE claimed no therapy responders for our independent data sets (Additional file 1: Table S1). In summary, our research could provide a new view for immune cell regulation mechanism and immunotherapy response prediction, and these models could be potential guidance for translational medicine and precision medicine. Moreover, we calculated different expression of biomarkers, which we claimed, between cell clusters (Additional file 9: Figure S8a). We found that these biomarkers different between singlecell types. We used CIBERSORT (https://cibersort.stanf ord.edu/) to perform deconvolution of cell types in bulk 
data GSE35640, and accessed immune score difference of CIRBERSORT cell type. We found that the cell difference of biomarkers in single cell level are connected with bulk data GSE35640, indicating a promising prospection of biomarkers we performed in precision medicine (Additional files 2, 3, 4, 5, 6, 7, 8).

\section{Conclusion}

We constructed regulation networks across immune single cell types and established that differently expressed genes between cells from responders and non-responders. In sum, 790 genes and 3048 interactions, including 26 receptors, 26 ligands, and 47 TFs were recognized by the regulation networks. Some differently expressed genes coincided with ligands and receptors in immune cell specific pathways. Further analysis of ligands and receptors in regulation networks proposed prediction biomarkers for inhibitor response classifier in metastatic melanoma with ICB therapy. We trained logistics regression models to test prediction accuracy of biomarkers for immunotherapy response. In summary, our study provided single cell-based regulation networks in context of melanoma model with or without ICB therapy and revealed several gene sets consist of therapy response biomarkers which is efficient for construction of ICB therapy response classifier. These results could provide a new view for immunotherapy response prediction, and these models could be potential guidance for translational medicine and precision medicine.

\section{Abbreviations}

ICB: Immune checkpoint blockade; PD-L1: Programmed death ligand 1;TF: Transcription factor; IL-2: Interleukin-2.

\section{Supplementary Information}

The online version contains supplementary material available at https://doi. org/10.1186/s12967-021-02962-8.

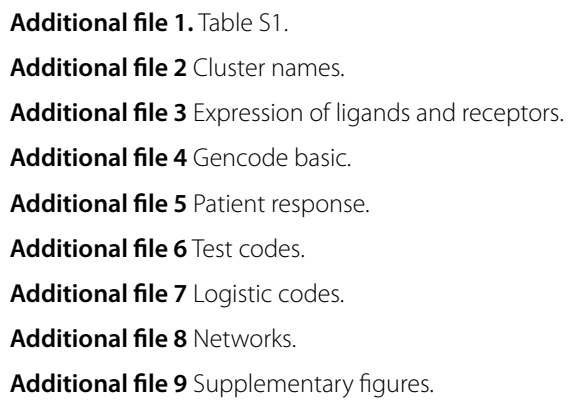

Acknowledgements

Not applicable.

\section{Authors' contributions}

Conceptualization: $\mathrm{XL}, \mathrm{YX}$ and $\mathrm{YZ}$; Data curation: JW, XZ and CZ; Formal analysis: JW and FL; Funding acquisition: XL; Investigation: JW and FL; Methodology:
JW and YX; Project administration: $Y Z$; Supervision: $Y X, X L$ and $Y Z$; Validation: $X Z, C Z, C H, Y X$ and $W M$; Visualization: JW and FL; Writing —original draft: JW; Writing - review \& editing: FL, YX, XL and YZ. All authors read and approved the final manuscript.

\section{Funding}

This work was supported by the National Key R\&D Program of China (2018YFC2000100), the National Natural Science Foundation of China (Grant Nos. 61873075, 31801107,31701145 and 61903105), the Heilongjiang Touyan Innovation Team Program, the China Postdoctoral Science Foundation (Grant No. 2019M661295), the China Postdoctoral Science Special Foundation (Grant No. 2020T130162), the Heilongjiang Postdoctoral Foundation (Grant No. LBH-Z19083) and the Fundamental Research Funds for the Provincial Universities (Grant No. 2018-KYYWF-0459), University Nursing Program for Young Scholars with Creative Talents in Heilongjiang Province Youth Science and technology innovation personnel training project [UNPYSCT-2017059], the Doctor Green Seedlings Breaking Ground Project of Harbin Medical University (Grant No.QMPT-2010).

\section{Availability of data and materials}

All data and codes in this study are available under proper request, please contact lixia@hrbmu.edu.cn. Our codes for AUC calculation on independent datasets are available in supplementary materials (Additional file 2, 3, 4, 5, 6, 7).

\section{Declarations}

Ethics approval and consent to participate

Not applicable.

\section{Consent for publication}

Not applicable.

\section{Competing interests}

The authors declare no conflict of interest.

Received: 16 November 2020 Accepted: 25 June 2021

Published online: 08 July 2021

References

1. Rebecca WW, Somasundaram R, Herlyn M. Pre-clinical modeling of cutaneous melanoma. Nat Commun. 2020;11(1):2858 (Epub 2020/06/07).

2. Gupta R, Janostiak R, Wajapeyee N. Transcriptional regulators and alterations that drive melanoma initiation and progression. Oncogene. 2020;39(48):7093-105 (Epub 2020/10/08).

3. Schumacher TN, Schreiber RD. Neoantigens in cancer immunotherapy. Science. 2015;348(6230):69-74 (Epub 2015/04/04).

4. Sharma P, Allison JP. The future of immune checkpoint therapy. Science. 2015;348(6230):56-61 (Epub 2015/04/04).

5. Sharma P, Allison JP. Immune checkpoint targeting in cancer therapy: toward combination strategies with curative potential. Cell. 2015;161(2):205-14 (Epub 2015/04/11).

6. Mellman I, Coukos G, Dranoff G. Cancer immunotherapy comes of age. Nature. 2011;480(7378):480-9 (Epub 2011/12/24).

7. Conway JR, Kofman E, Mo SS, Elmarakeby H, Van Allen E. Genomics of response to immune checkpoint therapies for cancer: implications for precision medicine. Genome Med. 2018;10(1):93 (Epub 2018/12/01).

8. Van Allen EM, Miao D, Schilling B, Shukla SA, Blank C, Zimmer L, et al. Genomic correlates of response to CTLA-4 blockade in metastatic melanoma. Science. 2015;350(6257):207-11 (Epub 2015/09/12).

9. Sade-Feldman M, Jiao YJ, Chen JH, Rooney MS, Barzily-Rokni M, Eliane JP, et al. Resistance to checkpoint blockade therapy through inactivation of antigen presentation. Nat Commun. 2017;8(1):1136 (Epub 2017/10/27).

10. Robert C, Schachter J, Long GV, Arance A, Grob JJ, Mortier L, et al. Pembrolizumab versus ipilimumab in advanced melanoma. N Engl J Med. 2015;372(26):2521-32 (Epub 2015/04/22).

11. Weber JS, D'Angelo SP, Minor D, Hodi FS, Gutzmer R, Neyns B, et al. Nivolumab versus chemotherapy in patients with advanced melanoma who progressed after anti-CTLA-4 treatment (CheckMate 037): 
a randomised, controlled, open-label, phase 3 trial. Lancet Oncol. 2015;16(4):375-84 (Epub 2015/03/22).

12. Herbst RS, Soria JC, Kowanetz M, Fine GD, Hamid O, Gordon MS, et al. Predictive correlates of response to the anti-PD-L1 antibody MPDL3280A in cancer patients. Nature. 2014;515(7528):563-7 (Epub 2014/11/28),

13. Finotello F, Rieder D, Hackl H, Trajanoski Z. Next-generation computational tools for interrogating cancer immunity. Nat Rev Genet. 2019;20(12):724-46 (Epub 2019/09/14)

14. Sade-Feldman M, Yizhak K, Bjorgaard SL, Ray JP, de Boer CG, Jenkins RW, et al. Defining $T$ cell states associated with response to checkpoint immunotherapy in melanoma. Cell. 2018;175(4):998-1013 (Epub 2018/11/06).

15. Tumeh PC, Harview CL, Yearley JH, Shintaku IP, Taylor EJ, Robert L, et al. PD-1 blockade induces responses by inhibiting adaptive immune resistance. Nature. 2014;515(7528):568-71 (Epub 2014/11/28)

16. Moreira A, Leisgang W, Schuler G, Heinzerling L. Eosinophilic count as a biomarker for prognosis of melanoma patients and its importance in the response to immunotherapy. Immunotherapy. 2017;9(2):115-21 (Epub 2017/01/28)

17. Tirosh I, Izar B, Prakadan SM, Wadsworth MH 2nd, Treacy D, Trombetta JJ, et al. Dissecting the multicellular ecosystem of metastatic melanoma by single-cell RNA-seq. Science. 2016;352(6282):189-96 (Epub 2016/04/29).

18. Demir E, Cary MP, Paley S, Fukuda K, Lemer C, Vastrik I, et al. The BioPAX community standard for pathway data sharing. Nat Biotechnol. 2010:28(9):935-42 (Epub 2010/09/11).

19. Ulloa-Montoya F, Louahed J, Dizier B, Gruselle O, Spiessens B, Lehmann $\mathrm{FF}$, et al. Predictive gene signature in MAGE-A3 antigen-specific cancer immunotherapy. J Clin Oncol. 2013;31 (19):2388-95 (Epub 2013/05/30).

20. Hu H, Miao YR, Jia LH, Yu QY, Zhang Q, Guo AY. AnimalTFDB 3.0: a comprehensive resource for annotation and prediction of animal transcription factors. Nucleic Acids Res. 2019;47(D1):D33-8 (Epub 2018/09/12).

21. Lizio M, Abugessaisa I, Noguchi S, Kondo A, Hasegawa A, Hon CC, et al. Update of the FANTOM web resource: expansion to provide additional transcriptome atlases. Nucleic Acids Res. 2019;47(D1):D752-8 (Epub 2018/11/09)

22. Barabasi AL, Albert R. Emergence of scaling in random networks. Science. 1999;286(5439):509-12 (Epub 1999/10/16).

23. Barabasi AL. Scale-free networks: a decade and beyond. Science. 2009;325(5939):412-3 (Epub 2009/07/25)

24. Zhang J, Liu L, Li J, Le TD. LncmiRSRN: identification and analysis of long non-coding RNA related miRNA sponge regulatory network in human cancer. Bioinformatics. 2018;34(24):4232-40 (Epub 2018/06/30).

25. Li J, Byrne KT, Yan F, Yamazoe T, Chen Z, Baslan T, et al. Tumor cell-intrinsic factors underlie heterogeneity of immune cell infiltration and response to immunotherapy. Immunity. 2018;49(1):178-93 (Epub 2018/07/01)

26. Li X, Wenes M, Romero P, Huang SC, Fendt SM, Ho PC. Navigating metabolic pathways to enhance antitumour immunity and immunotherapy. Nat Rev Clin Oncol. 2019;16(7):425-41 (Epub 2019/03/28).

27. Merhavi-Shoham E, Itzhaki O, Markel G, Schachter J, Besser MJ. Adoptive cell therapy for metastatic melanoma. Cancer J. 2017;23(1):48-53 (Epub 2017/01/24)

28. Weng J, Moriarty KE, Baio FE, Chu F, Kim SD, He J, et al. IL-15 enhances the antitumor effect of human antigen-specific CD8(+) T cells by cellular senescence delay. Oncoimmunology. 2016;5(12):e1237327 (Epub 2017/01/27)

29. Ercan G, Karlitepe A, Ozpolat B. Pancreatic cancer stem cells and therapeutic approaches. Anticancer Res. 2017;37(6):2761-75 (Epub 2017/05/30)

30. Yin T, Shi P, Gou S, Shen Q, Wang C. Dendritic cells loaded with pancreatic cancer stem cells (CSCS) lysates induce antitumor immune killing effect in vitro. PLoS ONE. 2014;9(12):e1 14581 (Epub 2014/12/19).

31. de Gast GC, Batchelor D, Kersten MJ, Vyth-Dreese FA, Sein J, van de Kasteele WF, et al. Temozolomide followed by combined immunotherapy with GM-CSF, low-dose IL2 and IFN alpha in patients with metastatic melanoma. Br J Cancer. 2003;88(2):175-80 (Epub 2003/03/01).

32. Harfuddin Z, Dharmadhikari B, Wong SC, Duan K, Poidinger M, Kwajah S, et al. Transcriptional and functional characterization of CD137L-dendritic cells identifies a novel dendritic cell phenotype. Sci Rep. 2016;6:29712.

33. Wang A, Chandran S, Shah SA, Chiu Y, Paria BC, Aghamolla T, et al. The stoichiometric production of IL-2 and IFN-gamma mRNA defines memory $T$ cells that can self-renew after adoptive transfer in humans. Sci Transl Med. 2012;4(149):149ra20 (Epub 2012/08/31)
34. Mi Y, Smith CC, Yang F, Qi Y, Roche KC, Serody JS, et al. A dual immunotherapy nanoparticle improves T-cell activation and cancer immunotherapy. Adv Mater. 2018;30(25):e1706098 (Epub 2018/04/25).

35. Martinez-Soria N, McKenzie L, Draper J, Ptasinska A, Issa H, Potluri S, et al. The oncogenic transcription factor RUNX1/ETO corrupts cell cycle regulation to drive leukemic transformation. Cancer Cell. 2019;35(4):705 (Epub 2019/04/17)

36. Hannun YA. Functions of ceramide in coordinating cellular responses to stress. Science. 1996;274(5294):1855-9 (Epub 1996/12/13).

37. Chou JJ, Li H, Salvesen GS, Yuan J, Wagner G. Solution structure of BID, an intracellular amplifier of apoptotic signaling. Cell. 1999;96(5):615-24 (Epub 1999/03/25).

38. Kimberlin DW. Neonatal herpes simplex infection. Clin Microbiol Rev. 2004;17(1):1-13 (Epub 2004/01/17).

39. Ogata H, Goto S, Sato K, Fujibuchi W, Bono H, Kanehisa M. KEGG: Kyoto encyclopedia of genes and genomes. Nucleic Acids Res. 1999;27(1):29-34 (Epub 1998/12/10).

40. Ashburner M, Ball CA, Blake JA, Botstein D, Butler H, Cherry JM, et al. Gene ontology: tool for the unification of biology The Gene Ontology Consortium. Nat Genet. 2000;25(1):25-9 (Epub 2000/05/10).

41. Seyednasrollah F, Rantanen K, Jaakkola P, Elo LL. ROTS: reproducible RNAseq biomarker detector-prognostic markers for clear cell renal cell cancer. Nucleic Acids Res. 2016;44(1):e1 (Epub 2015/08/13).

42. Gentles AJ, Newman AM, Liu CL, Bratman SV, Feng W, Kim D, et al. The prognostic landscape of genes and infiltrating immune cells across human cancers. Nat Med. 2015;21(8):938-45 (Epub 2015/07/21).

43. Niwa N, Tanaka N, Hongo H, Miyazaki Y, Takamatsu K, Mizuno R, et al. TNFAIP2 expression induces epithelial-to-mesenchymal transition and confers platinum resistance in urothelial cancer cells. Lab Invest. 2019;99(11):1702-13 (Epub 2019/07/03).

44. Yang $H$, Shao $R$, Huang $H$, Wang $X$, Rong $Z$, Lin $Y$. Engineering macrophages to phagocytose cancer cells by blocking the CD47/SIRPa axis. Cancer Med. 2019;8(9):4245-53 (Epub 2019/06/12).

45. Gu S, Ni T, Wang J, Liu Y, Fan Q, Wang Y, et al. CD47 blockade inhibits tumor progression through promoting phagocytosis of tumor cells by $\mathrm{M} 2$ polarized macrophages in endometrial cancer. J Immunol Res. 2018:2018:6156757 (Epub 2018/12/14)

46. Horrigan SK. Reproducibility Project: Cancer B Replication Study: The CD47-signal regulatory protein alpha (SIRPa) interaction is a therapeutic target for human solid tumors. Elife. 2017;6:e18173 (Epub 2017/01/20)

47. Fan Y, Si W, Ji W, Wang Z, Gao Z, Tian R, et al. Rack1 mediates Src binding to drug transporter P-glycoprotein and modulates its activity through regulating Caveolin-1 phosphorylation in breast cancer cells. Cell Death Dis. 2019;10(6):394 (Epub 2019/05/23).

48. Pereira PMR, Sharma SK, Carter LM, Edwards KJ, Pourat J, Ragupathi A, et al. Caveolin-1 mediates cellular distribution of HER2 and affects trastuzumab binding and therapeutic efficacy. Nat Commun. 2018;9(1):5137 (Epub 2018/12/05).

49. Ruffell B, Chang-Strachan D, Chan V, Rosenbusch A, Ho CM, Pryer N, et al. Macrophage IL-10 blocks CD8+T cell-dependent responses to chemotherapy by suppressing IL-12 expression in intratumoral dendritic cells. Cancer Cell. 2014;26(5):623-37 (Epub 2014/12/03).

50. Byron A, Humphries JD, Bass MD, Knight D, Humphries MJ. Proteomic analysis of integrin adhesion complexes. Sci Signal. 2011;4(167):pt2 (Epub 2011/04/07).

51. Sullivan RJ. Back to the future: rethinking and retooling IL2 in the immune checkpoint inhibitor era. Cancer Discov. 2019;9(6):694-5 (Epub 2019/06/05)

52. Abram $\mathrm{CL}$, Lowell $\mathrm{CA}$. The expanding role for ITAM-based signaling pathways in immune cells. Sci STKE. 2007;2007(377):re2 (Epub 2007/03/16).

53. Anderson DM, Maraskovsky E, Billingsley WL, Dougall WC, Tometsko ME, Roux ER, et al. A homologue of the TNF receptor and its ligand enhance T-cell growth and dendritic-cell function. Nature. 1997;390(6656):175-9 (Epub 1997/11/21).

54. Delgado ME, Brunner T. The many faces of tumor necrosis factor signaling in the intestinal epithelium. Genes Immun. 2019;20(8):609-26 (Epub 2019/01/29).

55. Cosset E, Ilmjarv S, Dutoit V, Elliott K, von Schalscha T, Camargo MF, et al. Glut3 addiction is a druggable vulnerability for a molecularly defined subpopulation of glioblastoma. Cancer Cell. 2017;32(6):856-68 (Epub 2017/12/05) 
56. Johnston AJ, Murphy KT, Jenkinson L, Laine D, Emmrich K, Faou P, et al. Targeting of Fn14 prevents cancer-induced cachexia and prolongs survival. Cell. 2015;162(6):1365-78 (Epub 2015/09/12).

57. Zanoni I, Ostuni R, Capuano G, Collini M, Caccia M, Ronchi AE, et al. CD14 regulates the dendritic cell life cycle after LPS exposure through NFAT activation. Nature. 2009;460(7252):264-8 (Epub 2009/06/16).

58. Praest $\mathrm{P}$, de Buhr $\mathrm{H}$, Wiertz E. A flow cytometry-based approach to unravel viral interference with the MHC class I antigen processing and presentation pathway. Methods Mol Biol. 2019;1988:187-98 (Epub 2019/05/31)

59. Kelly A, Trowsdale J. Genetics of antigen processing and presentation. Immunogenetics. 2019;71(3):161-70 (Epub 2018/09/15)

60. Nishino M, Ramaiya NH, Hatabu H, Hodi FS. Monitoring immune-checkpoint blockade: response evaluation and biomarker development. Nat Rev Clin Oncol. 2017;14(11):655-68 (Epub 2017/06/28).

61. Ayers M, Lunceford J, Nebozhyn M, Murphy E, Loboda A, Kaufman DR, et al. IFN-gamma-related mRNA profile predicts clinical response to PD-1 blockade. J Clin Invest. 2017;127(8):2930-40 (Epub 2017/06/27).
62. Tsai KK, Zarzoso I, Daud AI. PD-1 and PD-L1 antibodies for melanoma. Hum Vaccin Immunother. 2014;10(11):3111-6 (Epub 2015/01/28).

63. Kakavand H, Rawson RV, Pupo GM, Yang JYH, Menzies AM, Carlino MS, et al. PD-L1 expression and immune escape in melanoma resistance to MAPK inhibitors. Clin Cancer Res. 2017;23(20):6054-61 (Epub 2017/07/21)

64. Jiang P, Gu S, Pan D, Fu J, Sahu A, Hu X, et al. Signatures of T cell dysfunction and exclusion predict cancer immunotherapy response. Nat Med. 2018;24(10):1550-8 (Epub 2018/08/22).

\section{Publisher's Note}

Springer Nature remains neutral with regard to jurisdictional claims in published maps and institutional affiliations.
Ready to submit your research? Choose BMC and benefit from:

- fast, convenient online submission

- thorough peer review by experienced researchers in your field

- rapid publication on acceptance

- support for research data, including large and complex data types

- gold Open Access which fosters wider collaboration and increased citations

- maximum visibility for your research: over $100 \mathrm{M}$ website views per year

At BMC, research is always in progress.

Learn more biomedcentral.com/submissions 